\title{
CHARACTERIZATIONS OF TRIPOTENTS IN JB*-TRIPLES
}

\author{
REMO V. HÜGLI*
}

\begin{abstract}
The set $\mathscr{U}(A)$ of tripotents in a $\mathrm{JB}^{*}$-triple $A$ is characterized in various ways. Some of the characterizations use only the norm-structure of $A$. The partial order on $\mathscr{U}(A)$ as well as $\sigma$-finiteness of tripotents are described intrinsically in terms of the facial structure of the unit ball $A_{1}$ in $A$, i.e. without reference to the (pre-)dual of $A$. This extends similar results obtained in [6] and simplifies the metric characterization of partial isometries in $C^{*}$-algebras found in [1] (cf. [8]).
\end{abstract}

\section{Introduction}

In this article several conditions upon an element $a$ in a JB ${ }^{*}$-triple $A$, necessary and sufficient for $a$ to be a tripotent, i.e. that $\left\{\begin{array}{lll}a & a & a\end{array}\right\}=a$ are established. The results are based on the intricate connections that persist between the algebraic orthogonality on $A$ and the M-orthogonality, the former being defined in $\mathrm{JB}^{*}$-triples, the later in any normed vectorspace. Thereby we obtain a purely geometric description of the algebraic concept of tripotents. To be explicit, the M-complement $a^{\square}$ of the element $a$ in $A$ is defined to be the set of all elements $b$ in $A$ such that $\|a \pm b\|=\max \{\|a\|,\|b\|\}$. We show that an element $a$ of norm one in a $\mathrm{JB}^{*}$-triple $A$ is a tripotent if and only if

$$
a^{\square} \cap A_{1}=i a^{\square} \cap A_{1},
$$

where $A_{1}$ denotes the closed unit-ball of $A$ and $i$ the imaginary unit. This as well as further characterizations of tripotents are provided in Theorem 4.1.

The set $\mathscr{U}(A)$ of tripotents in $A$ is endowed with a partial order $\leq$ which is defined algebraically. It is shown that $(\mathscr{U}(A), \leq)$ is anti-order isomorphic to the partial order $(\mathscr{F} \mathscr{U}(A), \subseteq)$ of faces in $A_{1}$ generated by tripotents in $A$, ordered by set inclusion. The mapping $u \mapsto$ face $(u)$ is the corresponding anti-order isomorphism. This is shown in Theorem 4.4 which is a variation of results by Edwards and Rüttimann in [6] and [7], concerning the case of $\mathrm{JBW}^{*}$-triples and their pre-duals. Similar investigations were persued by Friedman and Russo,

\footnotetext{
* Supported in part by the Swiss National Science Foundation.

Received December 6, 2004; in revised form November 7, 2005.
} 
whose concept of facially symmetric spaces represents a geometric description of the pre-duals of $\mathrm{JBW}^{*}$-triples [11], [12]. Our description of $(\mathscr{U}(A), \leq)$ is completely intrinsic to $A_{1}$, i.e. it does not use any reference to the (pre-) dual of $A$, and it is valid for general $\mathrm{JB}^{*}$-triples. In Theorem 4.5 the results are applied to obtain also an intrinsic characterization of $\sigma$-finite tripotents.

The category of $\mathrm{JB}^{*}$-triples is strictly more general than those of some well known operator algebras, including $C^{*}$-algebras and $\mathrm{JB}^{*}$-algebras. The tripotents of a $C^{*}$-algebra are precisely its partial isometries. A metric characterization of the partial isometries in a $C^{*}$-algebra was provided earlier by Akeman and Weaver in [1]. Theorem 5.3 shows that their result can be seen as a special case of ours. For another proof of this result in complex as well as real JB*-triples we refer to the work by Fernandez-Polo, Martíinez Moreno and Peralta [8]. However, the explicit description by M-orthogonality has not been observed earlier.

The techniques used in this article are based on numerous works on JB*triples and $\mathrm{JBW}^{*}$-triples, in particular on [4], [6], [9], [11], [12], [13], [14], [16] and [17].

\section{Preliminaries}

Let $C$ be a convex subset in a vectorspace $E$. A convex subset $F$ of $C$ is said to be a face of $C$ if the following implications hold: If for some $t \in(0,1)$ and $a, b \in C$, the convex combination $t a+(1-t) b$ lies in $F$ then $a$ and $b$ themselves lie in $F$. Since the intersection of a family of faces of $C$ is also a face of $C$, for each subset $H$ of $C$, there exists the smallest face of $C$ containing $H$, denoted face ${ }_{C}(H)$ and referred to as the face of $C$ generated by $H$. Hence, the set $\mathscr{F}(C)$ of all faces of $C$, ordered by set inclusion, is a complete lattice with least element the empty set $\emptyset$ and largest element $C$. Let $\tau$ be a locally convex Hausdorff topology on $E$ and let $C$ be $\tau$-closed. A face $F$ of $C$ is said to be $\tau$-exposed if there exists a $\tau$-continuous linear functional $f$ on $E$ and a real number $t$ such that, for all elements $a$ in $C$,

$$
\operatorname{Re}(f(a))\left\{\begin{array}{ll}
=t & \text { if } a \in F \\
<t & \text { else }
\end{array} .\right.
$$

An arbitrary intersection of $\tau$-exposed faces is said to be a $\tau$-semi-exposed face of $C$. Let $\mathscr{F}_{\tau}(C), \mathscr{E}_{\tau}(C)$ and $\mathscr{S}_{\tau}(C)$ denote the sets of $\tau$-closed, $\tau$-exposed and $\tau$-semi-exposed faces of $C$ respectively. When ordered by set inclusion, $\mathscr{F}_{\tau}(C)$ and $\mathscr{S}_{\tau}(C)$ are complete lattices.

When $E$ is a normed vectorspace with dual space $E^{*}$ the abbreviations $n$ and $w^{*}$ will be used for the norm topology of $E$ and the weak* topology of $E^{*}$. For an element $a$ of $E_{1}$, define face $(a)$ to be the smallest face of $E_{1}$ which 
contains $a$. Let $H$ and $G$ be subsets of the unit ball $E_{1}$ in $E$ and of the unit ball $E_{1}^{*}$ of $E^{*}$ respectively. The sets $H^{\prime}$ and $G$, are defined by

$$
\begin{aligned}
& H^{\prime}=\left\{f \in E_{1}^{*}: f(a)=1 \forall a \in H\right\} ; \\
& G_{\prime}=\left\{a \in E_{1}: a(f)=1 \forall f \in G\right\} .
\end{aligned}
$$

Observe that $\left(H^{\prime}\right)$, is the least element of $\mathscr{S}_{n}\left(E_{1}\right)$ containing $H$, and $\left(G_{\prime}\right)^{\prime}$ is the least element of $\mathscr{S}_{w^{*}}\left(E_{1}^{*}\right)$ containing $G$. For more details, the reader is referred to [6], [7].

Two elements $a$ and $b$ of a normed vectorspace $E$ are said to be Morthogonal, denoted $a \square b$, if

$$
\|a \pm b\|=\max \{\|a\|,\|b\|\} .
$$

For a subset $H$ of the normed vectorspace $E$, the $M$-orthogonal complement (briefly the M-complement) $H^{\square}$ of $H$ is defined by

$$
H^{\square}=\{a \in E: a \square b, \forall b \in H\} .
$$

For a singleton set $\{a\}$ we write $a^{\square}$ instead of $\{a\}^{\square}$. Similarly, we write $a^{\prime}$ and $f$, if $a \in E$ and $f \in E^{*}$.

The M-complement is related to the facial structure of the unit-ball $E_{1}$ of $E$, as can be seen from straightforward considerations such as the following.

Proposition 2.1. Let a be an element in the closed unit ball $E_{1}$ of a normed vectorspace $E$. Then,

$$
a+\left(a^{\square} \cap E_{1}\right) \subseteq \text { face }(a)
$$

Proof. Consider an element $b$ in $a+\left(a^{\square} \cap E_{1}\right)$, that is $b=a+c$ for some $c \in\left(a^{\square} \cap E_{1}\right)$. Then $\|a \pm c\| \leq 1$. Hence both $a+c$ and $a-c$ lie in $E_{1}$. Since $a$ can be written as the convex combination

$$
a=\frac{1}{2}(a+c)+\frac{1}{2}(a-c),
$$

it follows that $b$ (and also $a-c$ ) lies in face $(a)$, as required.

The definitions of M-orthogonality and the M-complement make sense in real and complex normed vectorspaces. However, in the sequel we will assume $E$ to be complex. Denote by $S_{1}(\mathrm{C})$ and $S_{1}(E)$ the unit sphere in the complex plane $C$ and in $E$ and by $C_{1}$ the closed unit disc of C. The tangent disc $S_{a}$ and 
the flat tangent space $R_{a}$ corresponding to an element $a$ of $S_{1}(E)$ are defined by

$$
\begin{aligned}
S_{a} & =\left\{b \in E:\|a+s b\|=1 \forall s \in \mathrm{C}_{1}\right\}, \\
R_{a} & =\overline{\operatorname{lin}}_{\mathrm{C} S_{a}}{ }^{n} .
\end{aligned}
$$

The relations presented in the following lemma will be useful in subsequent considerations. They were proved in [4] Lemma 2.11.

LEMMA 2.2. Let a be an element of norm one in a complex normed vectorspace E. Then:

(i) $a^{\square} \cap E_{1}=\{b \in E:\|a+t b\|=1, \forall t \in[-1,1]\}$,

(ii) $i a^{\square} \cap a^{\square} \cap E_{1} \subseteq \sqrt{2} \cdot\left\{b \in E:\|a+z b\|=1 \forall z \in C_{1}\right\}$,

(iii) $i\left(a^{\square} \cap E_{1}\right)=(i a)^{\square} \cap E_{1}$,

(iv) $\operatorname{lin}_{\mathrm{R}}\left(i a^{\square} \cap a^{\square} \cap E_{1}\right)=\operatorname{lin}_{\mathrm{C}}\left(i a^{\square} \cap a^{\square} \cap E_{1}\right)$,

By (iii) the brackets in those expressions can be omitted. From (i) it is easially seen that $S_{a} \subseteq i a^{\square} \cap a^{\square} \cap E_{1}$. It follows from (ii) and (iv) that,

$$
\begin{aligned}
& S_{a} \subseteq i a^{\square} \cap a^{\square} \cap E_{1} \subseteq \sqrt{2} S_{a}, \\
& R_{a}=\varlimsup_{\operatorname{lin}_{C} i a^{\square} \cap a^{\square} \cap E_{1}}{ }^{n} .
\end{aligned}
$$

The case in which $E$ is a $\mathrm{JB}^{*}$-triple is the subject of the remaining sections.

\section{3. $\mathrm{JB}^{*}$-triples and $\mathrm{JBW}^{*}$-triples}

A Jordan ${ }^{*}$-triple is complex vectorspace $A$ equipped with a triple product $(a, b, c) \mapsto\{a b c\}$ from $A \times A \times A$ to $A$ which is symmetric and linear in the first and third variable, conjugate linear in the second variable and satisfies the Jordan triple identity

$$
[D(a, b), D(c, d)]=D(\{a b c\}, d)-D(c,\{d a b\}),
$$

where [, ] denotes the commutator, and $D(a, b)$ is the linear mapping on $A$ defined by $D(a, b) c=\{a b c\}$. A subspace $B$ of a $A$ is said to be a subtriple if $\left\{\begin{array}{lll}B & B & B\end{array}\right\}$ is contained in $B$.

A JB*-triple is a complex Banach space, which is a Jordan*-triple, and the triple product has the following properties. The mapping $(a, a) \mapsto D(a, a)$ is continuous from $A \times A$ to the Banach space $B(A)$ of bounded linear operators on $A$, for each element $a$ in $A, D(a, a)$ is hermitian in the sense of [2] Definition 5.1, with non-negative spectrum and has norm $\|D(a, a)\|=\|a\|^{2}$. If $A$ 
is also the dual of some Banach space $A_{*}$, then $A$ is said to be a $\mathrm{JBW}^{*}$-triple, and $A_{*}$ is referred to as the predual of $A$.

An important class of examples of $\mathrm{JB}^{*}$-triples is given by $\mathrm{C}^{*}$-algebras. [13]. When $A$ is a $\mathrm{C}^{*}$-algebra, the triple product is defined for $a, b, c \in A$, by

$$
\{a b c\}=\frac{1}{2}\left(a b^{*} c+c b^{*} a\right) .
$$

A JB*-triple behaves locally like a commutative $\mathrm{C}^{*}$-algebra, and an analogon of the $\mathrm{C}^{*}$-condition is valid, as can be seen from the next result. For proofs see [10] and [16].

Lemma 3.1. Let $A$ be $a \mathrm{JB}^{*}$-triple and let $a, b$ and $c$ be elements of $A$. Then, the following results hold.

(i) $\|\{a b c\}\| \leq\|a\|\|b\|\|c\|$.

(ii) $\left\|\left\{\begin{array}{lll}a & a & a\end{array}\right\}\right\|=\|a\|^{3}$.

(iii) The closed subtriple generated by an element a of $A$ is isometrically isomorphic as a Jordan*-triple to a commutative $C^{*}$-algebra.

A pair $a, b$ of elements of $A$ is said to be orthogonal, denoted $a \perp b$ if $D(a, b)$ is identically zero on $A$. It can be shown that this relation is symmetric. The algebraic annihilator $H^{\perp}$ of a non-empty subset $H$ of $A$ is defined to be the set

$$
H^{\perp}=\{a \in A: a \perp b \forall b \in H\}=\bigcap_{b \in H} b^{\perp} .
$$

Observe that $H^{\perp}$ is a norm closed subtriple of $A$, and $H^{\perp}$ is weak*-closed when $A$ is a $\mathrm{JBW}^{*}$-triple. As it was observed in [9] the properties described in Lemma 3.1 imply that the algebraic annihilator and the M-orthocomplement of $H$ are related by

$$
H^{\perp} \subseteq H^{\square}
$$

For any $a \in A$, define $a^{3}=\left\{\begin{array}{lll}a & a & a\end{array}\right\}$. Higher powers of $a$ can be defined unambiguously using the Jordan triple identity, by

$$
a^{2 n+1}=\left\{a \text { a } a^{2 n-1}\right\}=\left\{a a^{2 n-1} a\right\} .
$$

An element $u$ in $A$ is said to be a tripotent if $u^{3}=u$. The set of all tripotents of $A$ is denoted by $U(A)$. If $u$ and $v$ are tripotents of $A$ such that

$$
u \perp(v-u),
$$

then, $u$ is said to be less than or equal to $v$, denoted $u \leq v$. This relation provides a partial order on $\mathscr{U}(A)$ [17]. A tripotent $u$ in $A$ is $\sigma$-finite, if any 
set of pairwise orthogonal tripotents all of which are less than or equal to $u$ is of countable cardinality. The set of all $\sigma$-finite tripotents of $A$ is denoted by $\mathcal{U}_{\sigma}(A)$.

The partial order $(\mathscr{U}(A), \leq)$ has no largest element, except when $A$ is the null-vectorspace. Hence we may adjoin to $\mathscr{U}(A)$ an abstract largest element which we denote by $\omega$, and we define $\mathscr{U}(A)^{\sim}$ to be the set $\mathscr{U}(A) \cup\{\omega\}$. The investigations of the facial structure of $A_{1}$ carried out in [6] show that when $A$ is a $\mathrm{JBW}^{*}$-triple with predual $A_{*}$, then the sets $\mathscr{F}_{n}\left(A_{* 1}\right)$ and $\mathscr{E}_{n}\left(A_{* 1}\right)$ coincide, and also $\mathscr{F}_{w^{*}}\left(A_{1}\right)$ and $\mathscr{E}_{w^{*}}\left(A_{1}\right)$ coincide. Let the sets $\omega_{\text {, and }}\left(\omega_{1}\right)^{\prime}$ be defined by

$$
\omega_{1}=A_{* 1}, \quad\left(\omega_{1}\right)^{\prime}=\emptyset .
$$

This extends the mappings $G \mapsto G$, and $G \mapsto\left(G_{1}\right)^{\prime}$ to subsets of $\mathscr{U}(A)^{\sim}$. The next Lemma, which was obtained in [6], presents some profound connections between $\mathscr{F}_{n}\left(A_{* 1}\right), \mathscr{F}_{w^{*}}\left(A_{1}\right)$ and $\mathscr{U}(A)^{\sim}$.

Lemma 3.2. Let $A$ be a $\mathrm{JBW}^{*}$-triple with predual $A_{*}$. Then, the following results hold.

(i) The mapping $u \mapsto u$, is an order isomorphism from the partially ordered set $\mathscr{U}(A)^{\sim}$ of tripotents in $A$, with a largest element adjoined, onto the complete lattice $\mathscr{F}_{n}\left(A_{*, 1}\right)$ of all norm-closed faces of the closed unit ball $A_{*, 1}$ in $A_{*}$, and, hence, $\mathscr{U}(A)^{\sim}$ is a complete lattice.

(ii) The mapping $u \mapsto\left(u_{1}\right)^{\prime}$ is an anti-order-isomorphism from $\mathcal{U}(A)^{\sim}$ onto the complete lattice $\mathscr{F}_{w^{*}}\left(A_{1}\right)$ of weak ${ }^{*}$-closed faces of the closed unit ball $A_{1}$ in $A$ and

$$
\left(u_{1}\right)^{\prime}=u+\left(u^{\perp} \cap A_{1}\right) .
$$

The final result of this section connects the M-complement and the annihilator of subsets of $\mathcal{U}(A)$. A proof can be found in [4] Corollary 4.3.

LEMMA 3.3. Let $A$ be a $\mathrm{JB}^{*}$-triple and let $H$ be a non-empty subset of the set $\mathcal{U}(A)$ of tripotents in $A$. Then, the sets $H^{\square} \cap A_{1}$ and $H^{\perp} \cap A_{1}$ coincide.

\section{Characterizations of tripotents}

With the information presented so far, it is possible to establish the main results.

TheOREm 4.1. Let A be a $\mathrm{JB}^{*}$-triple and let a be an element in A of norm one. Then, the following conditions are equivalent.

(1) $a \in \mathscr{U}(A)$,

(2) $a^{\square} \cap A_{1}=a^{\perp} \cap A_{1}$, 
(3) $a^{\square} \cap A_{1} \subseteq a^{\perp} \cap A_{1}$,

(4) $a^{\square} \cap A_{1}=i a^{\square} \cap A_{1}$,

(5) $S_{a}=a^{\perp} \cap A_{1}$,

(6) $R_{a}=a^{\perp}$.

Proof. If $a$ is a tripotent of $A$, then (2) and (3) are immediate from Lemma 3.3. Notice that $a^{\perp}$ is a complex subspace of $A$, that $a^{\perp}=(i a)^{\perp}$, and that, by Lemma 2.2(iii), the sets $i\left(a^{\square} \cap A_{1}\right)$ and (ia $)^{\square} \cap A_{1}$ coincide. It follows from (2) that

$$
i a^{\square} \cap A_{1}=a^{\perp} \cap A_{1}=a^{\square} \cap A_{1} .
$$

This proves (4). To show (5), combine (2) with the relations (2.6) to obtain

$$
S_{a} \subseteq a^{\square} \cap i a^{\square} \cap A_{1}=a^{\perp} \cap A_{1} .
$$

For the reverse inclusion, let $b$ be any element of $a^{\perp} \cap A_{1}$. Since $a^{\perp}$ is a complex subspace of $A$, it follows from (2) that, for all $s \in S_{1}(\mathrm{C})$,

$$
s b \in a^{\perp} \cap A_{1}=a^{\square} \cap A_{1} .
$$

Therefore,

$$
\|a+s b\|=\max \{\|a\|,\|s b\|\}=1,
$$

that is, $b$ lies in $S_{a}$. This proves (5). The condition (6) is immediate from (5) by taking the closed linear span.

Suppose, that $a$ is not a tripotent. In order to disprove (2), (3) and (4), we need to find an element $b$ which lies in $a^{\square} \cap A_{1}$ but not in $a^{\perp} \cup i a^{\square}$. Similarly, we show hat there exists an element $c$ which lies in $S_{a}$ but not in $a^{\perp}$. By (2.6) and (2.7) this will disprove (5) and (6). The sought after elements $b$ and $c$ can be obtained from the spectral calculus, described in Lemma 3.1(iii). This is made explicit in the remainder of the proof.

Denote by $B$ the smallest norm-closed subtriple of $A$ containing $a$. By Lemma 3.1(ii), there exists a triple isomorphism $\varphi$ from $B$ onto the commutative $\mathrm{C}^{*}$-algebra $C_{0}(X)$ of continuous complex-valued functions on a locally compact subset $X$ of $[0,1]$ which vanish at zero. Observe that $\varphi(a)$ is equal to the function $\iota$ defined, for $t$ in $X$, by

$$
\iota(t)=t
$$

A function $f$ in $C_{0}(X)$ is a tripotent if and only if $f(X) \subseteq S_{1}(\mathrm{C}) \cup\{0\}$. The assumption that $a$ is not a tripotent implies that there exists an element $t_{0}$ in $X$ such that

$$
0<t_{0}<1 .
$$


Let $g$ be the element of $C_{0}(X)$ defined, for $t \in X$ by

$$
g(t)=\left\{\begin{array}{ll}
i \sqrt{1-t^{2}}\left(1-\frac{\left|t-t_{0}\right|}{1-t_{0}}\right), & \text { if }\left|t-t_{0}\right| \leq 1-t_{0} \\
0, & \text { if }\left|t-t_{0}\right|>1-t_{0}
\end{array} .\right.
$$

Clearly, $g$ has norm not greater than one in $C_{0}(X)$, i.e. $g$ lies in $C_{0}(X)_{1}$. Moreover, for all elements $t$ in $X$,

$$
|(\iota+g)(t)| \leq 1, \quad|(\iota-g)(t)| \leq 1,
$$

and, for $t=t_{0}$,

$$
\left|(\iota+g)\left(t_{0}\right)\right|=\left|(\iota-g)\left(t_{0}\right)\right|=1 .
$$

Therefore, $\iota+g$ and $\iota-g$ have norm one in $C_{0}(X)$, and, setting $b=\varphi^{-1}(g)$ entails

$$
\|a+b\|=\|a-b\|=\left\|\varphi^{-1}(\iota+g)\right\|=\sup _{t \in X}|t+g(t)|=1 .
$$

This shows that $a$ and $b$ are M-orthogonal. On the other hand,

$$
\|a-i b\| \geq\left|(\iota-i g)\left(t_{0}\right)\right|=\left|t_{0}-i g\left(t_{0}\right)\right|=t_{0}+\sqrt{1-t_{0}^{2}}>1 .
$$

Hence, $a$ and $\pm i b$ are not M-orthogonal, and $b$ is not contained in $i a^{\square}$. Furthermore, since $\varphi$ is a triple-isomorphism,

$$
\varphi\{b a a\}\left(t_{0}\right)=\{\varphi(b), \varphi(a), \varphi(a)\}\left(t_{0}\right)=\{g \iota \iota\}\left(t_{0}\right)=-i t_{0}^{2} \sqrt{1-t_{0}^{2}} \neq 0 .
$$

Therefore, $a$ and $b$ are not triple-orthogonal. We have shown that $b$ has the required properties.

Define the function $h$ in $C_{0}(X)$ by

$$
h(t)=\left\{\begin{array}{ll}
(1-t)\left(1-\frac{\left|t-t_{0}\right|}{1-t_{0}}\right), & \text { if }\left|t-t_{0}\right| \leq 1-t_{0} \\
0, & \text { if }\left|t-t_{0}\right|>1-t_{0}
\end{array} .\right.
$$

Also $h$ lies in $C_{0}(X)_{1}$, and for all $z$ in $C_{1}$ and all (positive) numbers $t$ with $\left|t-t_{0}\right| \leq 1-t_{0}$,

$$
\left|t+z(1-t)\left(1-\frac{\left|t-t_{0}\right|}{1-t_{0}}\right)\right| \leq 1
$$


The assumption that $a$ has norm one implies that $1 \in X$. It follows that, for all $z$ in $C_{1}$ and $t$ in $X$,

$$
\begin{aligned}
& |(\iota+z h)(t)| \leq|t+h(t)| \leq 1, \\
& |(\iota+z h)(1)|=1
\end{aligned}
$$

These relations show that $\iota+z h$ has norm one in $C_{0}(X)$, that is $h \in S_{l}$. Set $c=\varphi^{-1}(h)$. Then $c$ lies in $S_{a}$. On the other hand

$$
(\varphi\{c \quad a r\})\left(t_{0}\right)=\left(1-t_{0}\right) t_{0}^{2} \neq 0 .
$$

Therefore $c$ is not contained in $a^{\perp}$. This finishes the proof.

It is well known that two $\mathrm{JB}^{*}$-triples are triple isomorphic if and only if they are isometrically isomorphic as Banach spaces. We can use the above theorem to show that surjective linear isometries are algebraic isomorphisms. The converse can be proved using some arguments which are not directly connected with the methods considered here, and is therefore omitted. For original proofs the reader is referred to [14] Proposition 2.4 and [16] Proposition 5.5.

Corollary 4.2. Let $A$ and $B$ be JB*-triples, and let $\varphi: A \rightarrow B$ be a surjective linear isometry between $A$ and $B$. Then $\varphi$ is a triple isomorphism.

PROof. Let $a$ be an arbitrary element of $A$. Using polarisation of the triple product it can be seen that, for $a, b, c \in A$, there exist elements $\left(a_{k}\right)_{k=1}^{12}$ in $A$ and $\left(\alpha_{k}\right)_{k=1}^{12}$ in $\mathrm{C}$, such that

$$
\{a b c\}=\sum_{k=1}^{12} \alpha_{k} a_{k}^{3} .
$$

As next we show that $\varphi\left(a^{3}\right)=(\varphi(a))^{3}$, for all $a \in A$. The bi-adjoint $\varphi^{* *}$ of $\varphi$ is a weak* -continuous bijective isometry between $A^{* *}$ and $B^{* *}$. Morevoer, by [3], $A^{* *}$ and $B^{* *}$ are $\mathrm{JBW}^{*}$-triples, containing $A$ and $B$ as subtriples (even ideals) via the canonical embeddings. Therefore, $a$ can be regarded as an element of $A^{* *}$. By [9] there exists an orthogonal family $\left\{u_{j}\right\}_{j \in J}$ of tripotents in $A^{* *}$ and complex numbers $\left\{\alpha_{j}\right\}_{j \in K}$ such that

$$
a=\sum_{j \in J} \alpha_{j} u_{j}, \quad\|a\|=\sup _{j \in J}\left|\alpha_{j}\right| .
$$

The sum in this expression is weak*-convergent. Theorem 4.1(2) and Lemma 3.3 imply that $\left\{\varphi^{* *}\left(u_{j}\right)\right\}_{j \in J}$ is an orthogonal family in $\mathscr{U}\left(B^{* *}\right)$. It follows that

$$
\varphi^{* *}\left(a^{3}\right)=\varphi^{* *}\left(\sum_{j \in J} \alpha_{j}^{3} u_{j}\right)=\sum_{j \in J} \alpha_{j}^{3} \varphi^{* *}\left(u_{j}\right)=\left(\varphi^{* *}(a)\right)^{3} .
$$


By Lemma 3.1 and Equation (4.1) $\varphi$ is an injective triple homomorphism. This completes the proof.

In the remainder of this section we will be investigating the order structure of $\mathscr{U}(A)$. The following observation seems to have escaped notice so far.

Proposition 4.3. Let A be a $\mathrm{JBW}^{*}$-triple and let $u$ be a tripotent of $A$. Then,

$$
\operatorname{face}(u)=\left(u_{1}\right)^{\prime}=u+\left(u^{\perp} \cap A_{1}\right)=u+\left(u^{\square} \cap A_{1}\right) .
$$

In particular face $(u)$ is a weak ${ }^{*}$-closed subset of $A_{1}$.

Proof. Combine Lemma 3.2, Lemma 3.3 and Proposition 2.1 to obtain

$$
\text { face }(u) \subseteq u_{\prime}^{\prime}=u+\left(u^{\perp} \cap A_{1}\right)=u+\left(u^{\square} \cap A_{1}\right) \subseteq \text { face }(u) .
$$

Hence all of these sets coincide. Clearly $\left(u_{1}\right)^{\prime}$ is weak*-closed. This gives the proof.

Denote by $\mathscr{F} \mathscr{U}\left(A_{1}\right)$ the set of all faces of $A_{1}$ of the form face $(u)$, for some element $u$ in $\mathscr{U}(A)$. The above proposition and Lemma 3.2(ii) imply that

$$
\mathscr{F}_{w^{*}}\left(A_{1}\right)=\mathscr{F} \mathscr{U}\left(A_{1}\right) .
$$

As shown in the next theorem, the statement of Proposition 4.3 can be improved. It holds in $\mathrm{JB}^{*}$-triples and for the norm-semi-exposed face $\left(u^{\prime}\right)$, generated by $u$. This makes it possible to describe the order structure of $\mathcal{U}(A)$ in terms of the facial structure of $A_{1}$ without referring to the predual $A_{*}$.

THEOREM 4.4. Let A be a $\mathrm{JB}^{*}$-triple with $\mathscr{U}(A)$ the set of its tripotents and $\mathscr{F} \mathscr{U}(A)$ the set of those faces of $A_{1}$ which are generated by a tripotent. Then the map $u \mapsto$ face $(u)$ is an anti-order isomorphism between the partial orders $(\mathscr{U}(A), \leq)$ and $\left(\mathscr{F} \mathscr{U}\left(A_{1}\right), \subseteq\right)$. Moreover,

$$
\operatorname{face}(u)=\left(u^{\prime}\right),=u+\left(u^{\perp} \cap A_{1}\right)=u+\left(u^{\square} \cap A_{1}\right) .
$$

In particular, every face of $A_{1}$ generated by a tripotent is norm-closed.

When $A$ is a $\mathrm{JBW}^{*}$-triple, then $(\mathscr{F} \mathscr{U}(A) \cup \emptyset, \subseteq)$ is a complete lattice and is anti-order isomorphic to the lattice $\left(\mathscr{U}(A)^{\sim}, \leq\right)$.

Proof. When $A$ is a JB*-triple, let $j: A \rightarrow A^{* *}$ denote the canonical embedding into its second dual $A^{* *}$, a JBW ${ }^{*}$-triple with predual $A^{*}$ [3]. Consider a tripotent $u$ in $A$. Then $j(u) \in \mathscr{U}\left(A^{* *}\right)$. Observe that

$$
u \in u+\left(u^{\perp} \cap A_{1}\right)=u+\left(u^{\square} \cap A_{1}\right) \subseteq \text { face }_{A_{1}}(u) \subseteq\left(u^{\prime}\right), .
$$


If $b$ is an element of $\left(u^{\prime}\right)$, then by Proposition 4.3,

$$
j(b) \in\left(j(u)_{1}\right)^{\prime} \cap j(A)=j(u)+\left(j(u)^{\square} \cap A_{1}^{* *}\right) \cap j(A) .
$$

Hence $b$ lies in $u+\left(u^{\square} \cap A_{1}\right)$ which is therefore a superset of $\left(u^{\prime}\right)$,. It follows that

$$
u+\left(u^{\perp} \cap A_{1}\right)=u+\left(u^{\square} \cap A_{1}\right)=\text { face }_{A_{1}}(u)=\left(u^{\prime}\right), .
$$

The mapping $u \mapsto$ face $_{A_{1}}(u)$ from $\mathscr{U}(A)$ to $\mathscr{F} \mathscr{U}(A)$ is surjective by definition of $\mathscr{F} \mathscr{U}(A)$. To see that it is also injective, let $u$ and $v$ be a tripotents of $A$ such that face $_{A_{1}}(u)=$ face $_{A_{1}}(v)$. Then, by (4.4),

$$
j(u) \in j\left(\text { face }_{A_{1}}(v)\right) \subseteq j\left(\left(v^{\prime}\right)_{1}\right) \subseteq\left(j(v)_{1}\right)^{\prime} .
$$

Hence, $\left(j(u)_{1}\right)^{\prime} \subseteq\left(j(v)_{1}\right)^{\prime}$. In a similar way it is shown that $\left(j(v)_{1}\right)^{\prime} \subseteq\left(j(u)_{1}\right)^{\prime}$. Hence the equality $\left(j(u)_{1}\right)^{\prime}=\left(j(v)_{1}\right)^{\prime}$ holds. By Lemma 3.2(ii), $u$ equals $v$.

It remains to show that $u \mapsto$ face $_{A_{1}}(u)$ reverses the order structure. Suppose that $u, v \in \mathscr{U}(A)$ are such that $u \leq v$. Then there exists an element $w$ in $\mathscr{U}(A)$ with the properties $w \perp u$ and $v=u+w$. It follows that

$$
v \in u+\left(u^{\perp} \cap A_{1}\right)=u+u^{\square} \cap A_{1} \subseteq \text { face }_{A_{1}}(u) .
$$

This implies that

$$
\operatorname{face}_{A_{1}}(v) \subseteq \text { face }_{A_{1}}(u),
$$

as required.

In the case when $A$ is a JBW*-triple, it can be seen from Proposition 4.3 and the relations (4.5) that

$$
\operatorname{face}_{A_{1}}(u)=\left(u^{\prime}\right),=\left(u_{1}\right)^{\prime} .
$$

By Lemma 3.2, $u \mapsto$ face $_{A_{1}}$ is an anti-order isomorphims from the complete lattice $\left(\mathscr{U}(A)^{\sim}, \leq\right)$ to $\mathscr{F} \mathscr{U}(A)$. This finishes the proof.

It is now also possible to characterize $\sigma$-finiteness of tripotents in such a way that only the geometry of $A_{1}$ is used.

Theorem 4.5. Let $A$ be a $\mathrm{JB}^{*}$-triple. A tripotent $u$ of $A$ is $\sigma$-finite if and only if there are at most countably many elements $\left(a_{k}\right)_{k \in K}$ in the unit sphere $S_{1}(A)$ having the properties

(1) for all $k \in K, a_{k}^{\square} \cap A_{1}=i a_{k}^{\square} \cap A_{1}$,

(2) for $j \neq k, a_{k} \square a_{j}$,

(3) for all $k \in K$, face $(u) \subseteq$ face $\left(a_{k}\right)$. 
Proof. By Theorem 4.1, the condition (1) holds if and only if $\left\{a_{k}\right\}_{k \in K} \subseteq$ $\mathscr{U}(A)$. In this case, by Lemma 3.3, the relation $a_{j} \square a_{k}$ is equivalent to $a_{j} \perp a_{k}$. The prove is completed using (3) and Theorem 4.4.

\section{Applications to $\mathbf{C}^{*}$-algebras}

As it was shown in [13], any $\mathrm{C}^{*}$-algebra $A$ is a $\mathrm{JB}^{*}$-triple when equipped with the triple product given by (3.1).

The next lemma presents a well known fact. We include a proof for completeness.

Lemma 5.1. Let A be a $\mathrm{C}^{*}$-algebra, equipped with the triple product (3.1). Then, the set of tripotents of A coincides with that of the partial isometries.

Proof. Suppose that $u$ is a tripotent, i.e. $u=u u^{*} u$, then

$$
\left(u u^{*}\right)^{2}=\left(u u^{*} u\right) u^{*}=u u^{*} .
$$

Clearly, $u u^{*}$ is also self-adjoint. Hence $u$ is a partial isometry. Conversely, for each partial isometry $u$, the $\mathrm{C}^{*}$-condition implies that

$$
\begin{aligned}
\left\|u u^{*} u-u\right\|^{2} & =\left\|\left(u u^{*} u-u\right)\left(u u^{*} u-u\right)^{*}\right\| \\
& =\left\|u u^{*} u u^{*} u u^{*}-2\left(u u^{*}\right)^{2}-u u^{*}\right\|=0 .
\end{aligned}
$$

Hence, $u=u u^{*} u$, as required.

It is now obvious that we can provide a metric description of the partial isometries in $A$.

Theorem 5.2. A norm one element a of a $\mathrm{C}^{*}$-algbera $A$ is a partial isometry if and only if $a^{\square} \cap A_{1}=i a^{\square} \cap A_{1}$.

Proof. This is an immediate consequence of Theorem 4.1 and Lemma 5.1.

A metric description of partial isometries of $A$, different from that in Theorem 5.2 was found in [1]. Comparing those results with ours, we can show that the same description remains valid for tripotents of $\mathrm{JB}^{*}$-triples. For a norm-one element $a$ of $A$, consider the sets $X_{1}(a)$ and $X_{2}(a)$, defined by

$$
\begin{aligned}
& X_{1}(a)=\{b \in A: \exists r>0:\|a+r b\|=\|a-r b\|=1\}, \\
& X_{2}(a)=\{b \in A ; \forall z \in \mathrm{C} ;\|a+z b\|=\max \{1,\|z b\|\}\} .
\end{aligned}
$$

As shown in [1], $a$ is a partial isometry if and only if

$$
X_{1}(a)=X_{2}(a) .
$$


It is worth noting that the conditions $a^{\square} \cap A_{1}=i a^{\square} \cap A_{1}$ and $X_{1}(a)=X_{2}(a)$ are not equivalent in arbitrary complex normed vectorspaces. The question is wether this equivalence holds in $\mathrm{JB}^{*}$-triples. The affirmative answer, presented in the next theorem, provides yet another metric characterization of tripotents. An alternative proof and a generalization to real $\mathrm{JB}^{*}$-triples can be found in [8].

THEOREM 5.3. Let A be a $\mathrm{JB}^{*}$-triple, and let a be an element of norm one in $A$. Let the sets $X_{1}(a)$ and $X_{2}(a)$ be defined as in (5.1). Then a is a tripotent if and only if $X_{1}$ and $X_{2}$ coincide.

Proof. Suppose that $a \in \mathscr{U}(A)$. Observe that the inclusion $X_{2} \subseteq X_{1}$ is immediate from the definition of these sets. Hence we need only to show that $X_{1} \subseteq X_{2}$. Consider an element $b$ in $X_{1}$, i.e. there exists $r>0$ with $\|a \pm r b\|=1$. Then,

$$
2\|r b\|=\|a+r b-(a-r b)\| \leq\|a+r b\|+\|a-r b\|=2 .
$$

Hence $r b$ lies in $A_{1}$. Since $1=\max \{\|a\|,\|r b\|\}, r b$ lies also in $a^{\square}$. From this and Theorem 4.1(3), it follows that

$$
r b \in a^{\square} \cap A_{1}=a^{\perp} \cap A_{1} .
$$

The relation (3.2) implies that, for all $z \in \mathrm{C}$,

$$
z b=\frac{z}{r} r b \in \mathrm{C}\left(a^{\perp} \cap A_{1}\right)=a^{\perp} \subseteq a^{\square} .
$$

Therefore, $b$ lies in $X_{2}(a)$, as required.

Suppose that $X_{1}(a)=X_{2}(a)$, and consider an element $b$ in $a^{\square} \cap A_{1}$. From Lemma 2.2(i) it can be seen that $a^{\square} \cap A_{1} \subseteq X_{1}$. It follows that $b$ lies in $X_{2}(a)$. In particular

$$
\|a \pm i b\|=\max \{1,\|i b\|\} \leq 1 .
$$

This shows that $i b$ and $-i b$ are elements of $a^{\square} \cap A_{1}$. Hence $b$ lies in $i a^{\square} \cap A_{1}$. We conclude that $a^{\square} \cap A_{1} \subseteq i a^{\square} \cap A_{1}$. The reverse inclusion is obtained from similar arguments. By Theorem 4.1(4), $a$ is a tripotent. The proof is complete.

ACKNOWLedgements. Part of the main results presented in this article were obtained in the authors Ph.D. thesis [15]. He wishes to acknowledge the advise of the late G. T. Rüttimann, University of Berne, and of C. M. Edwards, University of Oxford, as well as the support received by H. Carnal, University of Berne. 


\section{REFERENCES}

1. Akeman, C., Weaver, N., Geometric characterizations of some classes of operators in $\mathrm{C}^{*}$ algebras and von Neumann algebras, Proc. Amer. Math. Soc 130, Nr. 10 (2002), 30333037.

2. Bonsall, F. F., Duncan, J., Numerical Ranges of Operators on Normed Spaces and of Elements of Normed Algebras, Cambridge Univ. Press, Cambridge 1971.

3. Dineen, S., The second dual of a $\mathrm{JB}^{*}$-triple, in Complex Analysis, Functional Analysis and Approximation theory, J. Mujica (ed.), North Holland, Amsterdam (1986), 67-69.

4. Edwards, C. M., Hügli, R. V., M-orthogonality and holomorphic rigidity in complex Banach spaces, Acta Sci. Math. (Szeged) 70 (2004), 237-264.

5. Edwards, C. M., Hügli, R. V., Order structure of GL-projections on complex Banach spaces, Preprint (2004).

6. Edwards, C. M., Rüttimann, G. T., On the facial structure of the unit balls in a JBW*-triple and its predual, J. London Math. Soc. 38 (1988), 317-332.

7. Edwards, C. M., Rüttimann, G. T. Orthogonal faces of the unit ball in a Banach space, Atti Sem. Mat. Fis. Univ. Modena 49 (2001), 473-493.

8. Fernández-Polo, F., Martíinez Moreno, J., Peralta, A. M., Geometric characterization of tripotents in real and complex JB*-triples, J. Math. AnalÄppl. 295, no. 2 (2004), 435-443.

9. Friedman, Y., Russo, B., Structure of the predual of a $\mathrm{JBW}^{*}$-triple, J. Reine Angew. Math. 356 (1985), 67-89.

10. Friedman, Y., Russo, B., The Gelfand-Naimark theorem for JB*-triples, Duke Math. J. 53 (1986), 139-148.

11. Friedman, Y., Russo, B., Affine structure of facially symmetric spaces, Math. Proc. Cambridge Philos. Soc. (1989) 106, 107-124.

12. Friedman, Y., Russo, B., Some affine geometric aspects of operator algebras, Pacific. J. Math. 137 No. 1 (1989), 123-144.

13. Harris, L. A., A generalisation of $C^{*}$-algebras, Proc. London Math. Soc. 42 (1981), 331-361.

14. Horn, G., Characterization of the predual and ideal structure of a $\mathrm{JBW}^{*}$-triple, Math. Scand. 61 (1983), 117-133.

15. Hügli, R. V., Contractive Projections on JBW*-Triples and their Predual, Inauguraldissertation Universität Bern, 2001.

16. Kaup, W., A Riemann mapping theorem for bounded symmetric domains, Math. Z. 183, 503-529.

17. Loos, O., Bounded Symmetric Domains and Jordan Pairs, Mathematical Lectures, University of California, Irvine, 1977.

18. Sakai, S., $C^{*}$-Algebras and $W^{*}$-Algebras, Springer, Berlin/Heidelberg/New York, 1971.

DEPT. OF MATHEMATICS

UNIVERSITY COLLEGE DUBLIN

BELFIELD

DUBLIN 4

IRELAND

E-mail: hugli@maths.ucd.ie 